\title{
The first six weeks - setting up a UK urgent dental care centre during the COVID-19 pandemic
}

Emily Carter ${ }^{1,2}$ Charlotte C. Currie, ${ }^{1,2}$ Abisola Asuni, ${ }^{1}$ Rachel Goldsmith, ${ }^{1}$ Grace Toon, ${ }^{1}$ Catherine Horridge, ${ }^{1}$ Sarah Simpson, ${ }^{1}$ Christopher Donnell, ${ }^{1}$ Mark Greenwood ${ }^{1,2}$ Graham Walton, ${ }^{1}$ Ben Cole, ${ }^{1,2}$ Justin Durham ${ }^{1,2}$ and Richard Holliday ${ }^{* 1,2}$

\section{Key points}

A summary is given of how urgent dental care was established in the North East of England during the COVID-19 pandemic, which may help with future preparedness for pandemics.
Aerosol generating procedures were almost always avoided in the delivery of urgent dental care.
A telephone triage system was effectively used to determine who needed clinical care and to separate symptomatic, asymptomatic and shielding patients, with very few failures in triage noted.

\begin{abstract}
Introduction The COVID-19 pandemic has posed many challenges, including provision of urgent dental care. This paper presents a prospective service evaluation during establishment of urgent dental care in the North East of England over a six-week period.
\end{abstract}

Aim To monitor patient volumes, demographics and outcomes at the North East urgent dental care centre and confirm appropriate care pathways.

Main outcome methods Data were collected on key characteristics of patients accessing urgent care from 23 March to 3 May 2020. Analysis was with descriptive statistics.

Results There were 1,746 patient triages (1,595 telephone and 151 face-to-face), resulting in 1,322 clinical consultations. The most common diagnoses were symptomatic irreversible pulpitis or apical periodontitis. Sixty-five percent of clinical consultations resulted in extractions and $0.8 \%$ in an aerosol generating procedure. Patients travelled $25 \mathrm{~km}$ on average to access care; however, this reduced as more urgent care centres were established. The majority of patients were asymptomatic of COVID-19 and, to our knowledge, no staff acquired infection due to occupational exposure.

Conclusion The urgent dental care centre effectively managed urgent and emergency dental care, with appropriate patient pathways established over the six-week period. Dental preparedness for future pandemic crises could be improved and informed by this data.

\section{Introduction}

The COVID-19 pandemic is likely to be one of the biggest challenges to face medicine and dentistry globally over the last century. During the week of 16 March 2020, the impact of COVID-19 began to affect dentistry in the UK. Primary dental care services were advised to radically reduce and subsequently stop routine

${ }^{1}$ Newcastle Hospitals NHS Foundation Trust, Newcastle upon Tyne, UK; ${ }^{2}$ School of Dental Sciences, Newcastle

University, Newcastle upon Tyne, UK.

${ }^{*}$ Correspondence to: Richard Holliday

Email address: richard.holliday@newcastle.ac.uk

Refereed Paper.

Accepted 15 May 2020

https://doi.org/10.1038/s41415-020-1708-2 dental care and patient face-to-face contact, and arrangements had to be made for the provision of care in urgent dental care centres (UDCCs) during the pandemic.

The Newcastle Dental Hospital (NDH) is part of the Newcastle upon Tyne Hospitals (NUTH) NHS Foundation Trust and serves a population of over three million. ${ }^{1}$ It delivers specialist dental services alongside undergraduate and postgraduate training and is fully integrated with the School of Dental Sciences at Newcastle University. The Dental Emergency Clinic and Child Dental Health Department at the NDH have been providing a walk-in clinic for many years, with the primary purpose of enabling clinical teaching for undergraduate dental students and training postgraduate dentists, while providing a valuable service for the local population.
During the pandemic, the dental emergency clinic was adapted to provide multidisciplinary seven-day emergency and urgent dental care ${ }^{2}$ to all age groups, and was staffed by qualified dentists from within the hospital, including consultants, specialists, speciality trainees and dental core trainees in oral surgery, restorative dentistry, paediatric dentistry, special care/ community dentistry and orthodontics, with support from oral and maxillofacial surgery consultants. At this early stage and in the absence of national guidance, the clinic had to balance providing a core service for the most in need (aiming to minimise dental attendances at medical emergency departments) against limiting patient throughput with a new, stricter triaging system to protect staff and other patients, and to preserve potentially limited personal protective equipment (PPE) supplies. 


\begin{abstract}
Fig. 1 Timeline of international (red), national (yellow) and local (blue) events in the COVID-19 pandemic (CMO = Chief Medical Officer, $\mathrm{CDO}=$ Chief Dental Officer, BAOS = British Assoication of Oral Surgeons, BAOMS = British Association of Oral and Maxillofacial Surgeons, WHO $=$ World Health Organisation)
\end{abstract}

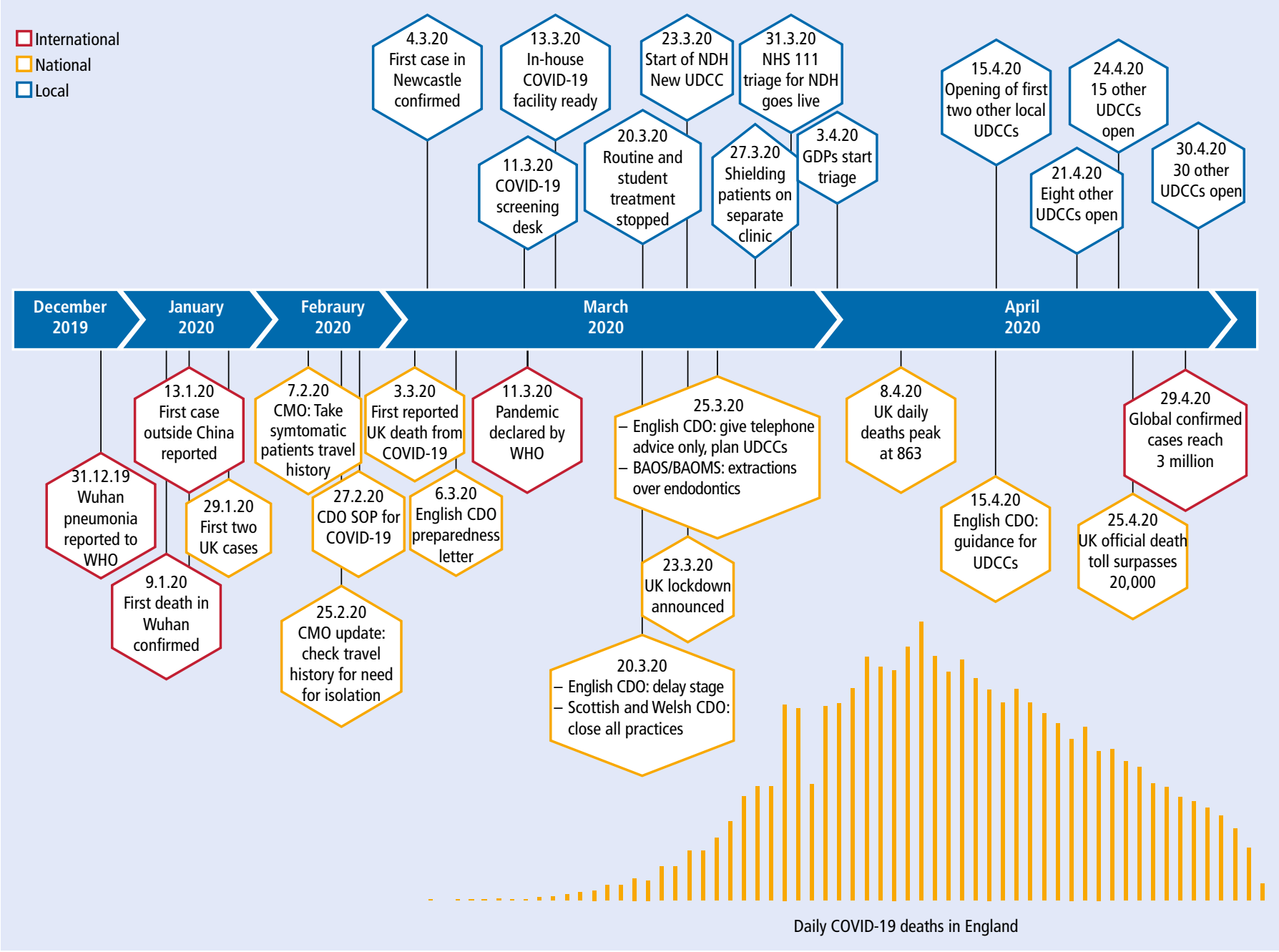

Following the inception of the adapted clinic on 23 March 2020, national guidance ${ }^{3}$ was published on 25 March stipulating that dental practices stop seeing patients face-to-face, leading to 337 NHS practices in our region closing their doors overnight. This guidance stated for patients to contact their general dental practitioner (GDP) for advice, analgesia and/or antimicrobials (commonly referred to as AAA) in the first instance, before any referral to a local UDCC to try and minimise patient travel and unnecessary attendances.

The urgent dental care service at the NDH evolved rapidly in response to challenges and changes in service and policy, while remaining open to the public. Figure 1 illustrates some key events in the COVID-19 pandemic alongside milestones in the development of the NDH UDCC. The clinic quickly transitioned to using remote triage for all patients, both in and outof-hours (OOH). In-hours (Monday-Friday,
8.30-16.30) triage was initially via walk-in and direct calls to the clinic from patients and dentists. With the assistance of NHS 111 , a national free-to-call non-emergency medical helpline, ${ }^{4}$ the in-hours service at $\mathrm{NDH}$ progressed to only accepting referrals by e-mail, mainly from GDPs and NHS 111 to manage the flow of referrals. A limited number of exceptions were made for clinically urgent walk-in cases who were triaged at a designated desk at the entrance of the building. All patients referred by e-mail had a further telephone triage by a dentist to establish their need for treatment and advice or an appointment was given. $\mathrm{OOH}$ triage (that is, weekends) was provided by NHS 111/Dental Clinical Assessment Service (DCAS). During triage, patients' COVID-19 status was also established by use of a symptom questionnaire. All patients attending the NDH UDCC had confirmation of their COVID-19 status and a temperature check on arrival. Both in-hours and $\mathrm{OOH}$, the service was divided into three distinct areas within the hospital: one for COVID-19 symptomatic and isolating patients; another for the asymptomatic; and a separate clinic for shielding and vulnerable patients (referred to as shielding in our unit) to minimise the risk of cross infection. The criteria for COVID-19 symptomatic patients was in line with NHS advice ${ }^{5}$ and we limited treatment in this group to true dental emergencies only (trauma, swelling or bleeding). The criteria for shielding patients is defined by the NHS as those who would be 'extremely vulnerable' if they were to contract COVID-19. These patients were grouped with those defined as 'vulnerable' under the general headings of: immunocompromised, severe respiratory disease, type I diabetes, pregnant, over the age of 70 and 'other'. Appropriate PPE was used for all staff in line with local and national guidance. . $^{6,8,9}$ 
Units from around the world have started to share their experiences of setting up similar UDCC services in their regions and healthcare settings. ${ }^{10,11}$ Throughout this pandemic, a service evaluation was conducted at the NDH UDCC. The aim of the service evaluation was to examine the volume of patient throughput, their demographics and outcomes in order to inform local decision-making and optimise appropriate care pathways. This paper presents our findings from the first six weeks, and is one of the first reports in the literature describing and exploring patient characterises and outcomes of those attending an UDCC during the COVID-19 pandemic.

\section{Methods}

A prospective service evaluation was carried out, which was registered on the NUTH Clinical Effectiveness Register (Ref. 10006) and approved before commencement. Data were collected on all patients attending the NDH UDCC, including paediatric, orthodontic and $\mathrm{OOH}$ care. Parameters recorded included: clinic attended, date of attendance, gender, date of birth, partial postcode, referral source, patient GDP registration status, triage type (telephone or face-to-face), triage and clinical consultation diagnoses and outcomes, COVID-19 status, and any repeat attendances at the UDCC. Ethnicity data were also collected retrospectively once it became apparent from media coverage that ethnicity may have an impact on COVID-19 prognosis. Anonymised data were collected by a selection of the UDCC clinicians (RH, AA, RG, GT, CH, SS, CD) using an Excel spreadsheet (Microsoft Office Professional Plus 2016, Version: 16.0.4993.1001) from 23 March to 3 May 2020. Aerosol generating procedures (AGPs) were defined as pulp extirpations or surgical extractions (using a handpiece) for the purpose of this service evaluation.

Data cleaning was completed both manually and in STATA release 13 (StataCorp LP, College Station, TX, USA). Where outcomes were identified as being missing, clinical notes were reviewed and data added retrospectively where available. Data were analysed in Statistical Package for the Social Sciences (Windows version 25.0.0.1; SPSS Inc., Chicago [IL], US) using descriptive statistics and exploratory analysis with chisquared tests. Geolytics (https://geo.sg/) was used to produce geographical location figures.
Fig. 2 Number of triage and clinical consultations by the NDH UDCC by week of service during the COVID-19 pandemic. Number of UDCCs open in the region shown for reference (yellow line). $00 \mathrm{H}$ triage consultations (provided by NHS 111) are not included as the data are unavailable. Week one starts on 23 March 2020 and week six ends on 3 May 2020

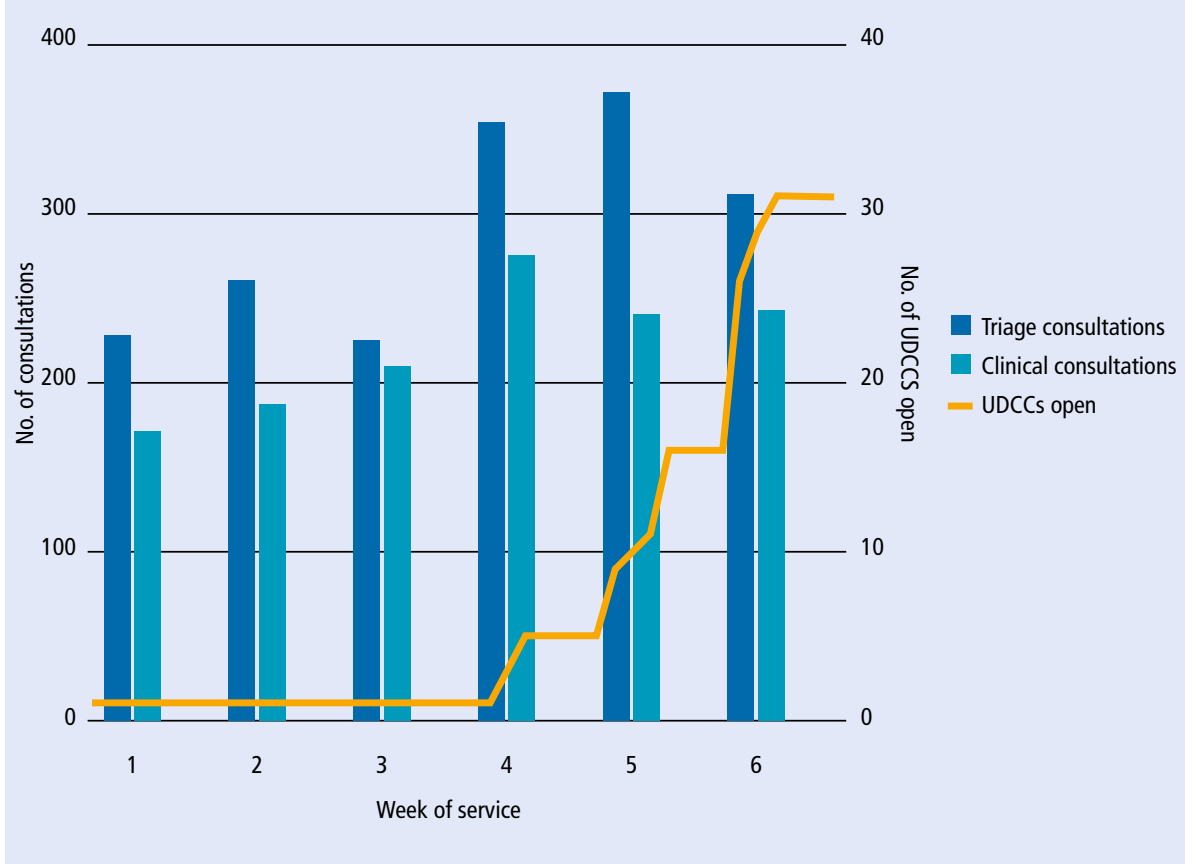

Estimated distance travelled was calculated using the fastest road route, as determined by ZIPCodeSoft (http://www.postcodedistance.com/distance-between-postcodes). Postcodes more than $210 \mathrm{~km}$ away were excluded from the 'distance travelled' analysis on the assumption that these people were not living in their primary residence during the pandemic.

\section{Results}

There were a total of 3,068 patient consultations at the UDCC over the six-week period (this equates to 1,595 telephone and 151 faceto-face triages, resulting in 1,322 clinical consultations). All clinical consultations were preceded by either a telephone or face-to-face triage. The majority of these consultations were for the in-hours adult service $(73 \%$, $\mathrm{n}=2,250)$, with $11 \%(\mathrm{n}=331)$ being for the in-hours paediatric service and $3 \%(n=102)$ for orthodontic services. Thirteen percent ( $\mathrm{n}=385 ; 90 \%$ [ $\mathrm{n}=347]$ adult, $10 \%[\mathrm{n}=38]$ paediatric) of consultations were in the $\mathrm{OOH}$ service $\mathrm{OOH}$ data includes face-to-face consultations only as triage was provided by an external provider, NHS 111/DCAS).

The distribution of the triage and clinical consultations over the six-week period is shown in Figure 2. The proportion of triage telephone consultations that resulted in a clinical visit increased during the first three weeks from $26 \%(n=37)$ in week one to $51 \%$ $(\mathrm{n}=112)$ in week three, before stabilising between $44-51 \%$ during weeks four to six. Face-to-face triage consultations (at the hospital reception desk) were most frequent in weeks one $(\mathrm{n}=87)$ and two $(\mathrm{n}=24)$, before reducing to very low levels in subsequent weeks (week three, $\mathrm{n}=6$; week four, $\mathrm{n}=17$; week five, $n=13$; week six, $n=4)$. The vast majority $(94 \%, n=142)$ of these face-to-face triage consultations at the hospital reception desk resulted in a clinical visit.

The number of clinical consultations increased from week one $(n=170)$ to week four ( $n=275)$, before stabilising in weeks five $(n=240)$ and six $(n=242)$. Both genders were equally represented in those accessing the service (50\% male and female). The mean age of those accessing the service was 36.5 years (range $0-89$, SD 19.1 years). In terms of patient ethnicity, $76 \%$ of patients attending clinical consultations were recorded as white British. Black, Asian and minority ethnic (BAME) made up 3.5\%, with 19\% being other, not known or not stated. Detailed patient demographics are summarised in the online supplementary information.

The geographical area served by the $\mathrm{NDH}$ UDCC over this period is illustrated in 

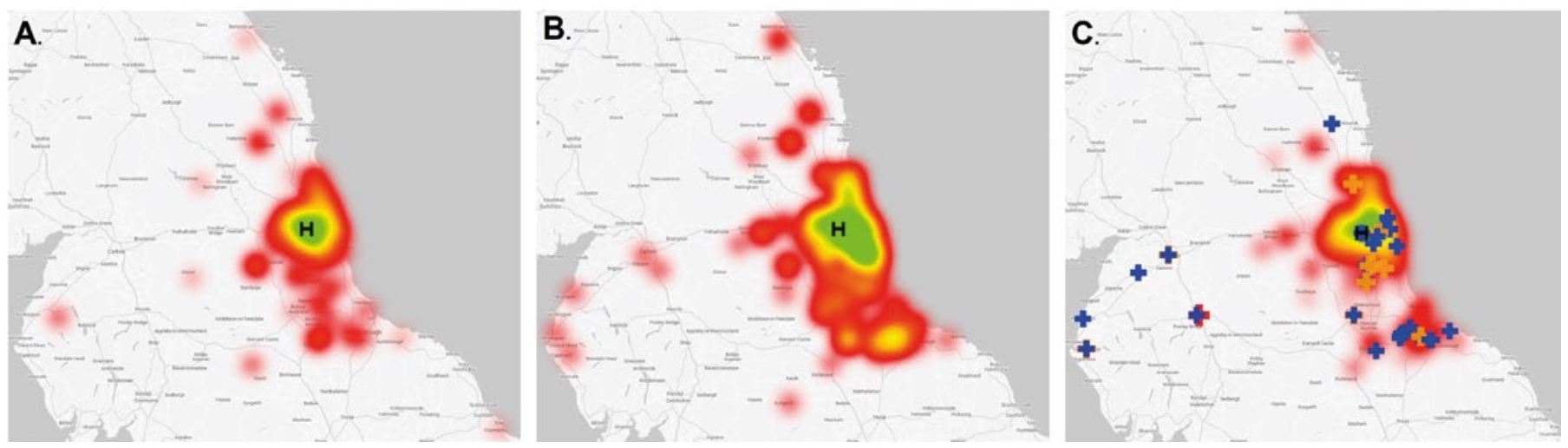

Fig. 3 Geographical area served by the NDH UDCC. Heat map indicates home address of those attending clinical visits in-hours (OOH data excluded as this service normally covers a wide region). Green = highest signal. Black ' $H$ ' illustrates the NDH UDCC location. a) Weeks one to two. b) Weeks three to four. c) Weeks five to six. Other UDCC locations shown in this panel by crosses, with blue indicating 'cold' sites, orange indicating 'warm' sites and 'red' indicating hot sites

Figure 3. Initially, a wide area was served, with patients travelling a mean of $22 \mathrm{~km}$ (range $0-188 \mathrm{~km}$, SD $26 \mathrm{~km})$ to access care during the first week, peaking at $29 \mathrm{~km}$ (range 3-206 $\mathrm{km}, \mathrm{SD} 26 \mathrm{~km}$ ) in week four and then reducing to $19 \mathrm{~km}$ (range 0-79 km, SD $16 \mathrm{~km}$ ) in week six. The weekly breakdowns are shown in the online supplementary information.

The majority of patients who accessed the service through the in-hours triage service reported having a $\operatorname{GDP}(81 \%, \mathrm{n}=1,417)$, and out of these, 1,126 (79\%) had attempted to contact them before contacting the UDCC, although this was lowest in the first week (59\%) (see online supplementary information). One hundred and thirty (7\%) had received no or inadequate advice or triage from their GDP (that is, provision of AAA, where appropriate), most frequently in the second week $(30 \%, \mathrm{n}=78)$; however, this markedly improved in later weeks (see online supplementary information). Two hundred and twelve (12\%) patients reported not having a GDP they saw regularly for treatment before the pandemic. There was a significant association between gender and having a GDP, with males significantly more likely to report that they did not have a GDP $\left(\mathrm{X}^{2}[1\right.$, $\mathrm{n}=1,628]=8.204, \mathrm{P}=0.004$ ).

The diagnoses of triage and clinical consultations is shown in Figure 4 (additional data including weekly breakdowns are provided in the online supplementary information). Most attendances were for acute pulpal and periapical complaints, which was consistent across adult, paediatric and $\mathrm{OOH}$ services. Symptomatic irreversible pulpitis was the most common triage diagnosis, whereas symptomatic apical periodontitis was the most common clinical diagnosis. The outcome of the clinical consultations is shown in Figure 5, with the majority resulting in extractions (63\%, $\mathrm{n}=832)$, of which four were surgical (two required a handpiece) and 17 (2\%) were incomplete (with follow-up arranged). Overall, only $0.8 \%(n=10)$ were AGPs (pulp extirpations or surgical extractions requiring use of a handpiece). Sixty-three percent of extracted teeth were molars, $15 \%$ premolars and $5 \%$ incisors/canines, with the remaining being primary teeth or multiple paediatric extractions.

Of the patients attending clinical consultations, and with a triage diagnosis indicating AAA was suitable, over half (56\%, $\mathrm{n}=245)$ had completed AAA, $35 \%(\mathrm{n}=153)$ had only received advice and analgesia advice but no antimicrobials, and 5\% $(\mathrm{n}=21)$ had received no advice. There was a gradual increase in the number of patients attending the clinical consultations with failed AAA, when the triage diagnosis suggested this was a suitable strategy, from $32 \%$ in week one to $70 \%$ in week four (full details in online supplementary information). For those with a triage diagnosis of symptomatic irreversible pulpitis and attending a clinical visit, $48 \%$ $(\mathrm{n}=183)$ had received AAA, 43\% $(\mathrm{n}=164)$ advice and analgesia advice, and $6 \%(\mathrm{n}=21)$ no advice.

The vast majority of patients attending the service were asymptomatic and nonshielding for COVID-19 (79\%, $\mathrm{n}=1,043)$, $15 \%(n=200)$ were shielding and $1 \%(n=17)$ were symptomatic. The proportion of shielding patients increased from a level of $2 \%(\mathrm{n}=4)$ in week one to a peak of $21 \%(n=57)$ in week four. Eight (0.5\%) of those triaged via the telephone as having a COVID-19 status of asymptomatic or shielding were subsequently deemed symptomatic and moved to the appropriate department or asked to go home and return after their isolation period. The weekly breakdowns and COVID-19 status of the triage consultations is shown in the online supplementary information.

The majority of patients attending clinical consultations were first-time attenders $(86 \%$, $\mathrm{n}=1,137)$, although $7 \%(\mathrm{n}=96)$ had previously attended during the COVID-19 pandemic and $5 \%(n=60)$ before the pandemic. Of those returning for repeat visits during the pandemic, the majority were for alveolar osteitis $(42 \%, \mathrm{n}=40)$ or for continuing acute pulpitic or periapical symptoms/diagnoses. The rate of patients returning with alveolar osteitis (40 cases/number of extraction-based procedures in the first five weeks) was $6 \%$.

\section{Discussion}

This service evaluation played an important role in the evolution and quality improvement of the NDH UDCC. It informed triage processes, staffing levels and provided useful feedback to our partners at the NHS 111 service, DCAS and GDPs. The data allowed the UDCC to use service information to inform decisions, alongside the guidance being produced. The data also give a unique opportunity to record and document urgent dental care demands when primary dental care services close, leaving only one provider, as was the case for the first three, almost four, weeks of this service. The insights from these data will help inform future pandemic preparedness planning.

Over the six-week period, there was a relatively equal split of patient gender 
Fig. 4 Diagnoses of a) triage and b) clinical consultations in the NDH UDCC during the COVID-19 pandemic. OOH triage consultations (provided by NHS 111) are not included as the data are unavailable. 'Other' notable diagnoses included: post-extraction pain (triage, $n=29$; clinical, $n=11$ ), removable prosthesis problems (triage, $n=3$; clinical, $n=1$ ) and extraoral non-odontogenic swelling (triage, $n=2 ;$ clinical, $\mathrm{n}=1$ )

a) triage

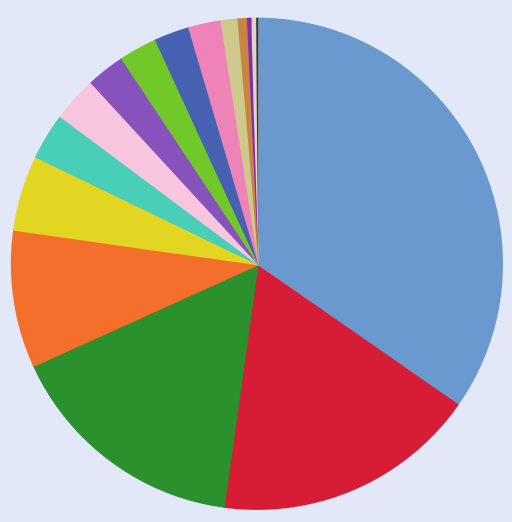

b) clinical consultations

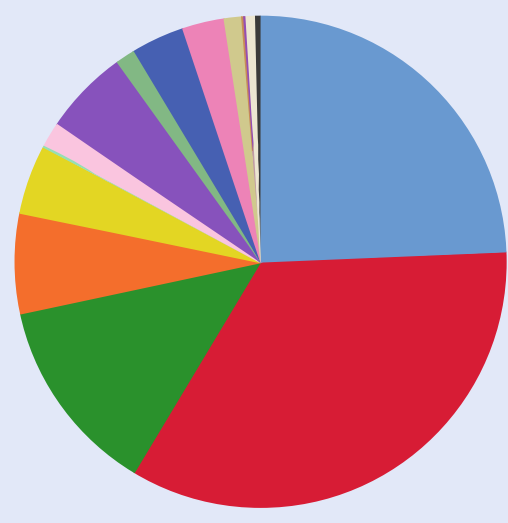

Symptomatic irreversible pulpitis

Symptomatic apical periodontitis

Acute apical abscess

Other

Avulsed, displaced or fractured teeth

Trauma from fractured or displaced orthodontic appliances Reversible pulpitis

- Acute periodontal abscess/Endo-perio lesions

Cracked, fractured, loose or displaced tooth fragments and restorations

Alveolar osteitis

Acute pericoronitis

Mobile teeth

Soft tissue lesion

NUG/P

TMD

Dentine hypersensitivity

Fig. 5 Outcomes of a) triage and b) clinical consultations in the NDH UDCC during the COVID-19 pandemic. For triage outcomes, those who were asked to call/attend other providers included $89 \%(n=93)$ to GDPs, $9 \%(n=9)$ to NHS 111 and $2 \%(n=2)$ to A\&E. Telephone advice constituted $85 \%(n=213)$ receiving analgesia advice, $8 \%(n=19)$ receiving trauma advice and $7 \%(n=18)$ advised temporary filling kit. For clinical outcomes, $3 \%(n=22)$ of the extraction outcomes had antimicrobials also prescribed, while $2 \%(n=17)$ were incomplete extractions, ie root or apex fracture, with follow-up arranged

a) triage

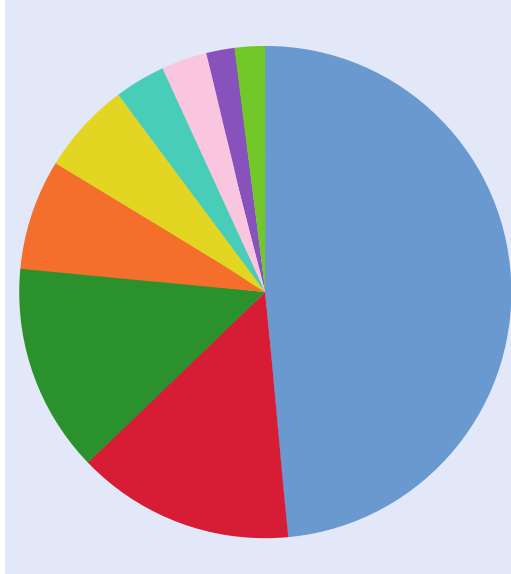

b) clinical consultations

Advised to come to NDH UDCC \& attended

- Telephone advice given

Other

Arranged/advised $A B s$ via GDP/GMP/pharmacist

Advised to call/attend other provider

Referred to another UDCC Pt declined treatment

Advised to come to NDH UDCC \& FTA

nP/RV post COVID

Orthodontic outcome

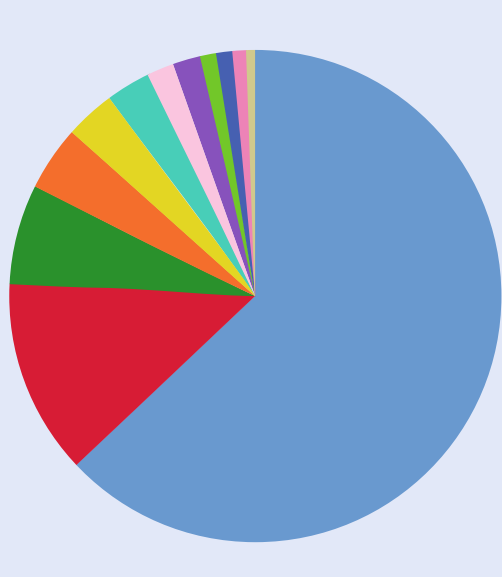

Extraction(s)

Prescribed ABs

Other

Dressing

- NDHXGA

- Trauma advice and/or treatment Irrigated with $\mathrm{CHX} /$ Saline

- Analgesia advice

I\&D

- Pt refused treatment

Returning for treatment/ review

Pulp extirpation attending the UDCC; this is in contrast with typical patient demographics accessing emergency dental care whereby the patient population is predominantly male. ${ }^{12,13,14}$ This change in demographic could reflect the change in service provision, whereby female patients who were more likely to be registered with a GDP in this cohort and who are more likely to seek care at an early stage ${ }^{15,16}$ started to present to the UDCC rather than primary care. The mean patient age was 37 years, which is in keeping with the literature of emergency dental care attenders who are typically in the third or fourth decade. ${ }^{12,13,14,17,18}$ The majority of patients attended with diagnoses of symptomatic irreversible pulpitis or apical periodontitis, which is again in keeping with the literature on emergency dental care. ${ }^{12,17,19,20,21}$

Over half of the patients seen for clinical consultation had followed national guidelines with regards to GDP triage and AAA provision, where this was appropriate, before attending. Use of antibiotics for dental pain during the pandemic is a controversial area, ${ }^{22}$ as UDCC attendances needed to be minimised, while also remaining aware of the potential for antibiotic resistance to increase with inappropriate use, the continued paucity of evidence to support their use in the management of acute localised painful dental conditions ${ }^{23}$ and the potential for adverse side effects. The number of patients reporting failure of AAA increased throughout the study period. This increase could represent more stringent enforcement of optimal AAA by our triage team before a clinical visit, or it could suggest that these measures aren't sufficient to manage severe dental pain. Advising use of antibiotics in the management of any acute dental problem remotely without the benefit of a clinical examination is challenging for clinical staff. Given the absence of clinical examination, it could risk inappropriate 
prescribing and thereby delivers a confusing public health message following several years of campaigning that 'antibiotics do not cure toothache.. ${ }^{24}$ However, given the requirement to immediately stop dental care and without arrangements in place for urgent care provision, the AAA strategy does seem to have been the only option in this initial stage, but could, with the benefit of hindsight, have been colloquialised as the $4 \mathrm{~A}$ approach - advice, analgesia and, if appropriate, antibiotics, thereby emphasising that antibiotics would only be appropriate in certain circumstances. Future preparedness planning should take this into account and hopefully avoid the need for a repeat of this situation.

Demand for the NDH UDCC service was high, with an initial peak of telephone calls immediately following the publication of the Chief Dental Officer for England's letter directing all primary care GDPs to give telephone advice only. ${ }^{3}$ This was followed by a continued high number of referrals to the service, which peaked in week five before declining as more UDCCs became operational across the region in weeks five and six. Due to the initial almost four-week period where NDH UDCC was the only service provider, patients accessed the service from a wide geographic area. This was clearly an undesirable situation given that UK government advice recommended only essential travel, avoidance of public transport where possible ${ }^{25}$ and the need for community-centred care to reduce flow into hospitals in order to limit the spread of infection. ${ }^{26}$ Throughout the period, we were liaising with our local commissioning team providing guidance for the setup of other UDCCs in the region. Clinical and practical advice was given to local providers setting up services, and included facilitating visits from practitioners setting up their clinics as UDCCs. In the final two weeks as more UDCCs were established, patient volumes decreased and patients became more local to the hospital, allowing a reduction in staffing level on the clinic and, in turn, the number of potential infectious contacts for staff members. This demand for the NDH UDCC in the early stages of the pandemic, due to lack of service provision regionally, demonstrates the need for early contingency planning in dentistry for future pandemics. Our data may inform the management of future changes to routine dental care during this pandemic, or the next.

Fortunately, the majority of patients attending the UDCC were asymptomatic for
COVID-19. This may be because symptomatic patients were following UK government guidelines and were self-isolating, too unwell with COVID-19 to consider dental pain a priority, or may also indicate the relatively low numbers of patients who coincidently had COVID-19 infection and require UDCC treatment. Our management of symptomatic or isolating patients was to try and avoid any attendance if at all possible, accepting only dental trauma, swelling or bleeding as reasons for attendance in this group, which would have reduced this number further. A challenging diagnostic dilemma was in the management of pyrexic patients attending with dental abscesses. In absence of further information, a cautious approach was utilised in this circumstance, as we had sufficient capacity and resource to do so.

The NDH UDCC was staffed by 69 clinicians and 55 dental nurses on a rotational basis. The in-hours adult urgent dental care service comprised 38 clinicians and 30 dental nurses. To the best of our knowledge, no staff-acquired infection occurred due to occupational exposure. We adopted the BAOS/BAOMS guidance advocating sessional use of FFP3 masks. ${ }^{6}$ Although this is beyond the level of PPE recommended by Public Health England, $\mathrm{NDH}$ felt that, initially, as the only provider of dentistry in the region, with staffing and patient footfall to match this, there was an increased risk of staff becoming infected and increasing the risk of nosocomial infection for patients and other staff. ${ }^{26}$ NUTH had good capacity for staff testing early on during the pandemic and have found that staff in patient-facing roles had a similar infection rate to those who work in administrative/back-of-house areas, suggesting community-acquired infection with COVID-19, rather than occupational, ${ }^{27}$ supporting the current management of PPE within the trust.

The most common treatment was extraction, with the majority of teeth being posterior teeth. This was in line with our local agreement to avoid AGPs unless essential. The presence of open dental pulps and oral sepsis is known to be more prevalent in lower socioeconomic groups ${ }^{28}$ and the area we serve includes some of the most deprived communities in England. ${ }^{29}$ This may account for a high extraction rate as a result of the extent of disease at presentation to the UDCC alongside our planned strategy of carrying out AGPs only when deemed necessary. Less than $1 \%$ of treatments required an AGP, which reflects our local agreement to avoid these unless essential. We only provided pulp extirpation when the tooth was strategically important and restorable, and we left fractured roots/ apices when there was no evidence of acute infection. Only $2 \%$ of extractions resulted in fractured roots/apices being left and these patients were given antibiotics where indicated, followed up by telephone, and either discharged if asymptomatic or put on a waiting list for surgical extraction after the pandemic if required. The presence of specialist oral surgeons at the UDCC may explain the low number of incomplete extractions as well as the low number of surgical extractions.

There are limitations of our data, which were collected as part of an ongoing service evaluation and hence in a pragmatic approach. There was no formal calibration of the data collectors; however, they were all experienced clinicians and were able to communicate easily within the team for any queries and consensus regarding data collection and input. The COVID-19 status data should be interpreted in the context of the testing limitations in the UK during this period (limited to symptomatic patients admitted to hospital or symptomatic key [health and social care] workers) and hence this status relied on the presence or recent history of symptoms. Data were collected from existing documentation and, on occasions, this has meant there are missing data in some cases. For the 'triage diagnosis', this was not always recorded and in this case the data collectors were asked to review the information recorded and use their judgement to give the most likely diagnosis (or reason for contact). Our anonymised data collection did not include a patient identification number, meaning we were unable to retrospectively state the exact number of individual patients seen within the service; rather, we report consultation episodes. The data were collected from a university teaching hospital and therefore may not be generalisable to primary care; however, during the first four weeks of data collection, the UDCC was the only centre providing urgent dental care throughout the North East of England and North Cumbria, and therefore reflect the real-life situation during the pandemic at that time. The data and experiences presented in this paper are our experience. They will be influenced by geographical, cultural and organisational factors, which need to be considered when generalising them to other locations and settings. 


\section{Conclusion}

In conclusion, our data give an insight into the patient characteristics, including urgent dental care requirements, of a lone regional provider during the COVID-19 pandemic. A telephone triage service was essential and demand grew over time. Acute pulpitic or periapical symptoms were most common, and most AGPs could be avoided. Very few symptomatic COVID-19 patients accessed the service, but demand from those shielding was high. Dental preparedness for this pandemic at national, regional and local levels was challenged and these data will help inform future planning.

\section{Conflict of interest}

The authors have no conflicts of interest to declare.

\section{Acknowledgements}

Richard Holliday is funded by a National Institute for Health Research ((NIHR)) Clinical Lectureship. Charlotte Currie is funded by an NIHR Doctoral Research Fellowship. The views expressed are those of the authors and not necessarily those of the NHS, the NIHR or the Department of Health and Social Care. Thanks to all the staff at the NDH for their support in managing the extensive service changes in this time, in particular but not exclusively, Lee Mercer, Kelly Gillan, Jenna Trainor, Phillipa Graham, Marie Allen, Jan McCalister, Andy Pike, Stewart Youngman and Tom Robson. We would also like to thank our local primary dental care colleagues who have been flexible and understanding during this challenging period.

\section{Author contributions}

E. Carter and C. Currie are joint first authors.

E. Carter, C. Currie and R Holliday contributed to the conception, design, data acquisition and interpretation, and drafted and critically revised the manuscript; J. Durham, G. Walton, B. Cole and $M$ Greenwood contributed to the data interpretation, and drafted and critically revised the manuscript; A. Asuni, R. Goldsmith, G. Toon, C. Horridge, S. Simpson and C Donnell contributed to the data acquisition, drafted and critically revised the manuscript.

\section{References}

1. Office for National Statistics. 2011 Census: Key Statistics and Quick Statistics for local authorities in the United Kingdom - Part 1. 2013. Available at https:// www.ons.gov.uk/peoplepopulationandcommunity/ populationandmigration/populationestimates/datasets/ 2011 censuskeystatisticsandquickstatisticsforlocalauthorities intheunitedkingdompart1 (accessed April 2020).

2. NHS England and NHS Improvement. 2019. Commissioning Standard for Urgent Dental Care. 2019. Available at https:// www.england.nhs.uk/wp-content/uploads/2019/07/com missioningstandardforurgentdental-care.pdf (accessed April 2020).

3. Chief Dental Officer. Issue 3: Preparedness for primary dental care. 2020. Available at https://www.england.nhs. uk/coronavirus/wp-content/uploads/sites/52/2020/03/ issue3preparednessletterforprimarydentalcare 25march2020.pdf (accessed April 2020).

4. National Health Service. NHS 111. 2017. Available online at https://www.nhs.uk/usingthenhs/nhs-services/ urgentandemergency-care/nhs-111/ (accessed April 2020).

5. National Health Service. Who's at higher risk from coronavirus. 2020. Available at https://www.nhs.uk/ conditions/coronaviruscovid19/peopleathigherriskfromcoronavirus/whosathigherriskfrom-coronavirus/ (accessed May 2020)

6. BAOS/BAOMS. BAOS and BAOMS joint position statement: Re-using FFP3 Masks and risk mitigation as we move from emergency to urgent care. 2020. Available at https://www. baos.org.uk/wp-content/uploads/2020/04/BAOS-BAOMS 22-April-2020-Statement-on-Re-using-FFP3-and-adviceas-we-move-from-emergency-to-urgent-care-provision.pdf (accessed May 2020).

7. BAOS/BAOMS. BAOS and BAOMS joint position statement: Updated COVID Advice from BAOMS and BAOS for our surgical teams. 2020. Available at https:// www.baos.org.uk/wp-content/uploads/2020/04/ BAOMS-and-BAOS-Advice-13-April-2020.pdf (accessed May 2020)

8. BAOS/BAOMS. BAOS and BAOMS joint position statement: Guidance PPE for patients with emergency oral and dental problems of unknown COVID Status. 2020. Available at https://www.baos.org.uk/wp-content/uploads/2020/03/ nowmBAOMSBAOSCOVIDAdviceUpdate24March-20191. pdf (accessed May 2020).

9. BAOS/BAOMS. BAOS and BAOMS joint position statement: BAOS \& BAOMS - Guidance for the care of OMFS and Oral Surgery patients where COVID is prevalent. 2020. Available at https://www.baos.org.uk/wp-content/uploads/2020/03/ BAOSBAOMSCovid19Postion-Paper.pdf (accessed May 2020)

10. Izzetti R, Nisi M, Gabriele F, Graziani F. COVID-19 transmission in dental practice: Brief review of preventive measures in Italy. J Dent Res 2020; DOI: 10.1177/0022034520920580.

11. Meng L, Hua F, Bian Z. Coronavirus disease 2019 (covid-19): Emerging and future challenges for dental and oral medicine. J Dent Res 2019; 99: 481-487.

12. Currie C C, Stone S J, Durham J. Pain and problems: a prospective cross-sectional study of the impact of dental emergencies. J Oral Rehabil 2015; 42: 883-889.

13. Scully C. The pattern of patient attendance for emergency care in a British dental teaching hospital. Community Dent Health 1995: 12: 151-154.
14. Anderson R, Thomas D W. 'Toothache stories': a qualitative investigation of why and how people seek emergency dental care. Community Dent Health 2003; 20: 106-111.

15. Dixon Woods M, Kirk K, Agarwal S et al. Vulnerable groups and access to health care: a critical interpretive review. 2005. Available at http://www.netscc.ac.uk/ hsdr/files/project/SDO_FR_081210025_V01.pdf (accessed March 2019).

16. Williams D R. The Health of Men: Structured Inequalities and Opportunities. Am J Public Health 2003; 93: 724-731.

17. Matthews R, Scully C, Porter K, Griffiths M. An analysis of conditions presenting to a dental hospital emergency clinic. Health Trends 1992; 24: 126-128.

18. Nayee $S$, Kutty $S$, Akintola D. Patient attendance at a UK dental hospital emergency clinic. Br Dent J 2015; 219: 485-488.

19. Gibson G B, Blasberg B, Hill S J. A prospective survey of hospital ambulatory dental emergencies. Part 1: Patient and emergency characteristics. Spec Care Dent 1993; 13: $61-65$.

20. Matthews R W, Peak J D, Scully C. The efficacy of management of acute dental pain. Br Dent J 1994; 176: 413-416.

21. Tramini P, Al Qadi Nassar B, Valcarcel J, Gibert P. Factors associated with the use of emergency dental care facilities in a French public hospital. Spec Care Dent 2010; 30: 66-71.

22. Faculty of General Dental Practice. Open letter on prescribing antibiotics during COVID-19. 2020. Available at https://www.fgdp.org.uk/news/openletterprescribingan tibioticsduringcovid19-\%C2\%A0 (accessed May 2020).

23. Tampi $\mathrm{M}$, Pilcher $\mathrm{L}$, Urquhart $\mathrm{O}$ et al. Antibiotics for the urgent management of symptomatic irreversible pulpitis, symptomatic apical periodontitis, and localized acute apical abscess: Systematic review and meta-analysis-a report of the American Dental Association. J Am Dent Assoc 2019; DOI: 10.1016/j.adaj.2019.09.011.

24. Public Health England. Dental antimicrobial stewardship: toolkit. 2016. Available online at https://www.gov. uk/guidance/dentalantimicrobialstewardship-toolkit (accessed May 2020).

25. UK Government. Coronavirus (COVID-19): UK transport and travel advice. 2020. Available online at https:// www.gov.uk/guidance/coronaviruscovid19uktransportan dtravel-advice (accessed April 2020)

26. Nacoti M, Ciocca A, Giupponi A, Brambillasca P, Lussana F, Pisano M. At the Epicentre of the Covid-19 pandemic and humanitarian crises in Italy: Changing perspective on preparation and mitigation. Catalyst 2020; 1: 1-5.

27. Hunter E, Price D, Murphy E et al. First experience of COVID-19 screening of healthcare workers in England. Lancet 2020; DOI: 10.1016/S0140-6736(20)30970-3.

28. Steele J, Pitts N, Fuller E, Treasure E. 3: Urgent Conditions - a report from the Adult Dental Health Survey 2009. 2011. Available at https://files.digital. nhs.uk/publicationimport/pub01xxx/pub01086/ aduldenthealsurvsummthemthe32009rep5.pdf (accessed May 2020).

29. Ministry of Housing, Communities \& Local Government. The English Indices of Deprivation 2019. 2019. Available at https://assets.publishing.service.gov.uk/government/ uploads/system/uploads/attachment_data/file/833947/ loD2019_Research_Report.pdf (accessed May 2020). 